\title{
Síndrome de Charles Bonnet. Manejo con haloperidol en paciente nonagenaria. Caso clínico
}

\author{
Camila Valencia, José G abriel Franco \\ Charle Bonnet syndrome. Report of \\ one case managed with haloperidol
}

Charles Bonnet syndrome is an underrecognized condition characterized by complex visual hallucinations, ocular problems causing visual deterioration and preserved cognitive status. Its prevalence is $5 / 1000$ in ambulatory ophthalmologic patients. Generally occurs in elderly people in whom it may be confused with delirium or dementia. The first management step is to improve vision, if possible. Hallucinations may be managed pharmacologically. We report a 94 year-old woman with the characteristic clinical picture of the syndrome that improved with haloperidol (Rev Méd Chile 2008; 136: 347-50).

(Key w ords: Charles Bonnet syndrome; Delirium; Hallucinations; Haloperidol)

Recibido el 26 de abril, 2007. Aceptado el 19 de junio, 2007.

Grupo de Investigación en Psiquiatría de Enlace, Facultad de Medicina, Universidad Pontificia Bolivariana. Medellín, Colombia.

$\mathrm{E}$ ste síndrome fue descrito por el filósofo Crancés Charles Bonnet en 1760, quien hablaba de las alucinaciones visuales formadas que presentaba su abuelo, que tenía buena salud general y reducción de la agudeza visual por cataratas $^{1}$. Ocurre generalmente en ancianos con enfermedades visuales, como glaucoma, cataratas y degeneración macular ${ }^{2-5}$. La prevalencia varía según la población estudiada, se estima que es de 5 por cada 1.000 pacientes oftalmológicos ambula-

Correspondencia a: Dra. Camila Valencia. Diagonal 79A \#5-299 Bloque 6 Apartamento 122, Medellín-Colombia. E mail: camival3@hotmail.com torios y se considera que en muchas oportunidades es pasado por alto ${ }^{6}$.

Se caracteriza por alucinaciones visuales vívidas, complejas, estereotipadas, que pueden tener movimiento, empeoran con la fatiga, estrés y baja iluminación ${ }^{4,5,7}$. Se han establecido los siguientes criterios diagnósticos $\left.{ }^{4,6,7}: 1\right)$ Alucinaciones visuales formadas, complejas, persistentes. 2) Conciencia parcial o total de que las alucinaciones son irreales. 3) Ausencia de alucinaciones en otras modalidades sensoriales. 4) Ausencia de delirios primarios o secundarios. La evolución puede ser: episódica (de días a meses); periódica (con remisión durante meses) o continua ${ }^{8}$.

La etiología es desconocida, se habla de deaferentación del sistema visual, que puede afectar los campos receptivos en la corteza visual, 
ocasionando descargas neuronales espontáneas y percepciones sensoriales anormales 2,5 .

En ancianos, es importante diferenciar el síndrome de Charles Bonnet (SCB) de entidades que pueden presentar alucinaciones, en especial: delirium, demencia y alucinosis pedunculares. Otras entidades que pueden ser relevantes en el diagnóstico diferencial son: trastornos afectivos, migraña, esquizofrenia, epilepsia y alucinaciones asociadas al sueño ${ }^{4}$.

En cuanto al manejo, se debe corregir la alteración visual de base $e^{5,7}$. Las alucinaciones suelen desaparecer al solucionar la causa o con la ceguera total. Cuando no es posible corregir la causa o si a pesar de corregirla continúan los cambios en la sensopercepción, debe hacerse un manejo sintomático para reducir o eliminar las alucinaciones y controlar la ansiedad asociada. Aunque no hay un tratamiento aceptado universalmente, entre las opciones farmacológicas más reportadas están los anticonvulsivantes (carbamazepina, ácido valproico, gabapentin) y la olanzapina ${ }^{4,7,8}$. Algunos pacientes han mejorado con haloperidol, mientras que, según algunas revisiones, en otros ha tenido un efecto limitado $7,9,10$. El tratamiento debe incluir además psicoeducación, reducción del aislamiento, estímulos (música, televisión) y mejor iluminación ambiental ${ }^{4,7}$.

Presentamos este caso característico del SCB con el fin de contribuir al reconocimiento de esta condición y de proponer una opción de manejo en pacientes muy ancianos con comorbilidad médica.

Caso clínico. Mujer de 94 años, evaluada por psiquiatría, a petición de medicina interna, por posible delirium. Con 4 años de evolución de percepciones visuales complejas, vívidas, descritas como imágenes de "hombres con sombrero y llamaradas", que aparecían a cualquier hora. El inicio fue insidioso, para luego establecerse un curso episódico/periódico con intervalos de remisiones y exacerbaciones de 2 a 4 meses de duración. Su reacción ante dichas percepciones era de marcada ansiedad e inquietud, reconocía que estas imágenes no eran reales y además ni ella ni su cuidador las asociaban a otras alteraciones en el estado de ánimo o de la conciencia, tampoco a cambios del comportamiento, ni desorientación.
La paciente era hipertensa, con enfermedad renal crónica (en hemodiálisis desde 2002). Manifestaba disminución progresiva de la agudeza visual de unos 6 años de evolución. Sin antecedentes personales o familiares, de tipo psiquiátrico o neurológico.

Al examen mental se encontraba alerta, orientada, euproséxica, sin alteraciones del movimiento. Pensamiento de curso normal, sin ideación delirante, con alucinaciones visuales, "hombres con sombrero y llamaradas", que reconocía como irreales. Sin alucinaciones auditivas. Lenguaje normal. Afecto modulado, sin cogniciones depresivas. Juicio y raciocinio normales.

Según la versión colombiana del Miniexamen Cognoscitivo (mini-mental) ${ }^{11}$, no presentaba deterioro cognitivo (puntuación de 22, normal para su edad y escolaridad). También se descartó delirium por medio de la versión colombiana de la Escala Revisada-98 para Valoración del Delirium (DRSR98), en la que obtuvo una puntuación de 6 (rango normal: 0-14) ${ }^{12}$. El examen neurológico era normal, excepto por el evidente déficit visual. Se inició haloperidol a dosis de $0,5 \mathrm{mg}$ por noche, se aumentó gradualmente hasta $2 \mathrm{mg}$ por noche (20 gotas) y se hizo psicoeducación sobre el síndrome. Las alucinaciones desaparecieron a la semana de iniciado el fármaco, sin observarse, hasta el presente, efectos extrapiramidales.

La evaluación oftalmológica encontró agudeza visual de cuenta dedos a un metro, en ambos ojos y atrofia irreversible bilateral del nervio óptico, para la cual no fue posible esclarecer etiología. Se decidió entonces continuar el haloperidol. Cuando la paciente suspendió el medicamento por su cuenta durante varias semanas, reaparecieron las alucinaciones, las cuales desaparecieron al reiniciarlo (2 mg por noche). Al momento de hacer esta comunicación lleva 6 meses en tratamiento.

\section{DisCUSIÓN}

El SCB puede ocurrir a cualquier edad, en personas con deterioro visual de cualquier origen ${ }^{2-4}$. Cuando hay buena conciencia de la irrealidad de las percepciones, éstas deben diferenciarse clínicamente de las alucinosis pedunculares, que son alucinaciones visuales sin objeto y con conciencia de irrealidad, acompañadas de signos originados 
en el tallo cerebral, aparecen al caer la tarde, sin producir ansiedad y ocurren en ancianos sin deterioro visual, generalmente como consecuencia de afecciones vasculares cerebrales, entre otras causas $^{13}$.

El síndrome puede causar ansiedad, enojo, irritabilidad, inquietud motora, llanto y comportamientos que pueden hacer que con frecuencia sea confundido con delirium, demencia o cualquier forma de psicosis; en especial, afectivas $5,7,14$. Un error diagnóstico de este tipo en un paciente muy anciano, podría llevar a la conclusión errónea de que lo único que hay para ofrecer son cuidados paliativos o institucionalización.

La evidencia sobre la eficacia de los medicamentos en el SCB proviene de estudios no controlados con pocos pacientes y de reportes de casos. En esta paciente, el manejo sintomático fue con haloperidol a dosis bajas, debido a los riesgos de somnolencia y alteraciones metabólicas asociadas al uso de anticonvulsivantes u olanzapina en personas muy ancianas y con comorbilidades ${ }^{15}$. El haloperidol ha sido usado con seguridad en pacientes ancianos y críticamente enfermos desde hace años; cuando se administra en forma de gotas su titulación es más fácil que con otros fármacos. Con su uso es importante evaluar al paciente,

\section{REFERENCIAS}

1. Hedges TR. Charles Bonnet, his life and his syndrome. Surv Ophthalmol 2007; 52: 111-14.

2. FFyтche DH. Visual hallucinations and the Charles Bonnet Syndrome. Curr Psychiatry Rep 2005; 7: 168-79.

3. Shiraishi Y, Terao T, Ibi K, Nakamura J, Tawara A. Charles Bonnet Syndrome and visual acuity-the involvement of dynamic or acute sensory deprivation. Eur Arch Psychiatry Clin Neurosci 2004; 254: 362-4.

4. Menon GJ, Rahman I, Menon SJ, Dutton GN. Complex Visual Hallucinations in the visually impaired: The Charles Bonnet Syndrome. Surv Ophthal 2003; 48: 58-72.

5. ROvNER BW. The Charles Bonnet Syndrome: a review of recent research. Curr Opin Ophthalmol 2006; 17: 275-7. especialmente las primeras semanas, para vigilar la aparición de efectos extrapiramidales ${ }^{15}$.

Al revisar 2 reportes de caso citados en la literatura $^{7}$, como ejemplo del efecto limitado del haloperidol en el manejo de este síndrome, se encontró que en uno de ellos la dosis de haloperidol fue probablemente baja, a pesar de lo cual sí hubo mejoría. Dicho reporte es del caso de una mujer de 69 años con antecedentes de diabetes mellitus e hipertensa que presentaba reducción de la agudeza visual asociada a cataratas, hemorragia vítrea y glaucoma, fue tratada con $1 \mathrm{mg}$ diario de haloperidol con reducción de sus alucinaciones hasta una frecuencia de aparición cada 4 días $^{16}$. El otro caso es el de un hombre de 73 años con degeneración macular y antecedente de trastorno depresivo mayor en tratamiento antidepresivo que mejoró totalmente de sus alucinaciones visuales con haloperidol (dosis no reportada) ${ }^{17}$.

En conclusión, la anamnesis y exploración física pueden ayudar a diferenciar el SCB de otras entidades de peor pronóstico. El haloperidol podría ser una opción de manejo sintomático de este síndrome en pacientes muy ancianos o con contraindicación para otros psicofármacos, pero para corroborar esto son necesarios estudios controlados.

6. Shiraishi Y, Terao T, Ibi K, Nakamura J, Tawara A. The rarity of Charles Bonnet syndrome. J Psychiatr Res 2004; 38: 207-13.

7. Eperjesi F, Akbarali N. Rehabilitation in Charles Bonnet syndrome: a review of treatment options. Clin Exp Optom 2004; 87: 149-52.

8. Coletti MM, Milano E, Gasverde S, Gianelli M, Giordana MT. Olanzapine therapy in hallucinatory visions related to Bonnet syndrome. Neurol Sci 2005; 26: 168-70.

9. Batra A, Bartels M, Wormstall H. Therapeutic options in Charles Bonnet syndrome. Acta psychiatr Scand 1997; 96: 129-33.

10. Komreich C, Dan B, Verbanck P, Pelc I. Treating Charles Bonnet syndrome: understanding inconcistence. J Clin Psychofarmacol 2000; 20: 396.

11. Rosselli D, Ardila A, Pradilla G, Morillo L, Bautista L, Rey O et al. El examen mental abreviado (Mini-Mental State Examination) como 
prueba de selección para el diagnóstico de demencia: estudio poblacional colombiano. Rev Neurol 2000; 30: 425-32.

12. Franco JG, Mejía MA, Ochoa SB, Ramírez LF, Bulbena A, Trzepacz PT. Escala revisada-98 para valoración del delirium (DRS-R98): Adaptación colombiana de la versión española. Actas Esp Psiquiatr 2007; (en prensa).

13. Lanteri-Laura G. Las alucinaciones. México DF: Fondo de cultura económica, 1994.

14. González M, De Pablo J, Valdés M. Delirium, la confusión de los clínicos. Rev Méd Chil 2003; 131: 1051-60.
15. Janicak PG, Davis JM, Preskorn SH, Ayd FJ. Principles and practice of psychopharmacotherapy. $3^{\text {rd }}$ edition. Philadelphia: Lippincott Williams \& Wilkins, 2001.

16. Chen J, Gómez M, Veit S, O’Dowd MA. Visual hallucinations in a blinded elderly woman: Charles Bonnet syndrome, an underrecognized clinical condition. Gen Hosp Psychiatry 1996; 18 : 453-5.

17. Fernández A, Lichtshein G, Vieweg WVR, Winfrey CJ. Charles Bonnet syndrome with peripheral and central findings. Int J Geriatr Psychiat 1996; 11 : 773-8. 